\title{
A Rare Case of Hemophagocytic Lymphohistiocytosis with Concurrent Extra-Pulmonary Sarcoidosis Presenting as Fever of Unknown Origin
}

\author{
Dharti Patel*, Kinza Muzzaffar, Ateeq Mubarik, Nirali Vassa, Salman Muddassir
}

*Corresponding author: Dharti Patel

Address: Oak Hill Hospital Brooksville, FL 34613, USA

e-mail $\bowtie$ dharti45@gmail.com

Received: 21 November 2019; Accepted: 26 November 2019

\begin{abstract}
Hemophagocytic Lymphistiocytosis (HLH) is a rare syndrome with an uncontrolled inflammation due to an ineffective immune response to antigens which leads to multiorgan failure, if not treated aggressively (Atteritano et al., 2012). HLH can be genetic or acquired. The genetic form which is a primary HLH diagnosed at an early age, is associated with primary immunodeficiency syndromes (Rosado and Kim, 2013), and the acquired form, which can occur at any age, have various etiologies including infections, malignancies or rheumatological diseases (Schram and Berliner, 2015; Sawhney et al., 2001). Histiocyte Society defines the diagnostic criteria for HLH on the basis of few characteristics including fever, cytopenias, splenomegaly, hyperferritinemia, hypertriglyceridemia, decreased fibrinogen, hemophagocytosis, increased IL-2 receptor alpha and decreased natural killer (NK) function. The clinical course of the disease is very aggressive with the mortality rates from $8 \%$ to $22 \%$, so early diagnosis is the key to early treatment. Sarcoidosis is a chronic granulomatous but also a multisystem inflammatory disease which is rarely associated with sarcoidosis (Abughanimeh et al., 2018). In order to add our contribution to the available literature on this under recognized association, we report a unique case of a 70 year old female, presented with fever of unknown origin who was newly diagnosed with sarcoidosis and was complicated by HLH later in the hospital course.
\end{abstract}

Key Words: Hemophagocytic lymphohistiocytosis, Macrophage activation, Cytokine storm, Immune dysregulation, Phagocytosis

\section{Introduction}

Hemophagocytic Lymphohistiocytosis (HLH) is a rare syndrome with uncontrolled inflammation due to an ineffective immune response to antigens, leading to multiorgan failure if not treated aggressively (Atteritano et al., 2012). HLH can be genetic or acquired. Primary HLH, which is diagnosed at an early age, is associated with immunodeficiency syndromes (Rosado and Kim, 2013).The acquired form, which can occur at any age, has various etiologies including infections, malignancies or rheumatological diseases (Schram and Berliner, 2015; Sawhney et al., 2001). The Histiocyte Society defines the diagnostic criteria for HLH on the basis of few characteristics including fever, cytopenia, splenomegaly, hyperferritinemia, hypertriglyceridemia, decreased fibrinogen, hemophagocytosis, increased IL-2 receptor alpha and decreased natural killer (NK) function. Sarcoidosis is a chronic granulomatous and 
multisystem inflammatory disease, which is rarely associated with HLH (Sawhney et al., 2001). The rheumatologic form of secondary HLH is also known as macrophage activation syndrome (MAS). The most common rheumatic diseases associated with HLH are juvenile arthritis and systemic lupus erythematosus (SLE). The clinical course of the disease is very aggressive, with the mortality rates ranging from $8 \%$ to $22 \%$, therefore early diagnosis is key to initiation of prompt treatment (Abughanimeh et al., 2018). In order to add our contribution to the available literature on this under recognized association, we report a unique case of a 70 year old female who presented with fever of unknown origin and ultimately was diagnosed with sarcoidosis complicated by HLH during her hospital course.

\section{Case Presentation}

A 70 year old Hispanic woman, with a past medical history of hypothyroidism and GERD, presented to our hospital for the evaluation of fever of unknown origin, diarrhea,and dark urine. She was previously hospitalized for three weeks in Puerto Rico with the same complaints, undergoing various tests and imaging, and sent home with no real diagnosis. She presented to the hospital as she was still experiencing quotidian fevers with associated weakness. Upon admission, she was febrile and pancytopenic with white blood count (WBC) ranging from 2.o to 3.2, platelet count varying from 24 to 117, and hemoglobin $(\mathrm{Hgb})$ ranging from 6.8-8.7. Multiple sources of infection were detected including an extended spectrum beta lactamase (ESBL) E. coli urinary tract infection (UTI) and Campylobacter detected in a stool sample. Despite management with antibiotics, she continued to experience daily fevers. Later, a bone marrow biopsy was also done to determine the underlying cause of pancytopenia, which revealed a non caseating granuloma. The angiotensin converting enzyme (ACE) level was also elevated and the diagnosis of Sarcoidosis was made. After the initiation of corticosteroids, she showed clinical improvement and was discharged after 3.5 weeks of inpatient care with a high dose of prednisone. She followed hematology and oncology outpatient and eventually the dose of prednisone was decreased by half. However, at this time she became symptomatic again, experiencing generalized weakness and fevers.

Thirteen days later, she returned to the hospital after a fall due to weakness and altered mental status. She was found to be febrile and severely pancytopenic with WBC ranging from o.61.8, platelets 10-38 and $\mathrm{Hgb}$ ranging from 6.9-9. Her total bilirubin was four times more than her last measured total bilirubin on the previous admission. Additional labs included aspartate transferase (AST) $295 \mathrm{IU} / \mathrm{L}$, alanine transferase (ALT) of $81 \mathrm{IU} / \mathrm{L}$, lactate dehydrogenase (LDH) 
of $625 \mathrm{U} / \mathrm{l}$, alkaline phosphatase (ALP) of $219 \mathrm{IU} / \mathrm{L}$, ferritin of 77,449 ng/mL, elevated D-dimer of $5.8 \mathrm{mg} / \mathrm{L}$, with normal fibrinogen levels. Imaging revealed splenomegaly and nodular cirrhotic liver. High dose of intravenous corticosteroids was resumed and she was started on granix for pancytopenia, procrit for underlying undiagnosed CKD, continuous blood transfusions for low $\mathrm{Hgb}$, and broad spectrum antibiotics. Given worsening pancytopenia, high ferritin and liver dysfunction, Macrophage Activation Syndrome (MAS) was suspected. Repeat bone marrow biopsy showed normal hematopoiesis replaced by proliferation of histiocytes and noncaseating granulomas. CD23 was negative but CD68 was positive indicating histiocytes within the granulomas. As she met five out of the eight criteria for hemophagocytic lymphohistiocytosis, etoposide was initiated. Within the next couple days, she developed encephalopathy and went into multisystem organ failure. Due to her worsening clinical status and minimal response to etoposide, the family decided to withdraw care and she passed away.

\section{Discussion}

Hemophagocytic lymphohistiocytosis is a rare disease that results from uncontrolled proliferation of activated lymphocytes and histiocytes, which secretes high amounts of inflammatory cytokines, leading to eventual multi-organ failure if not treated aggressively. The prevalence of this disease is reported as $3 \%$ in association with rheumatologic disease, with only seven known cases of HLH associated with sarcoidosis (Abughanimeh et al., 2018).

Commonly associated cardinal signs and symptoms include prolonged fever, neurological abnormalities such as seizures and altered mental status. Characteristic biochemical markers include pancytopenia, abnormally high liver function tests, elevated triglycerides and ferritin with low fibrinogen.

The pathogenesis of HLH involves dysregulation of T cell activity, causing increased cytokine release such as interleukin (IL)-1, IL-6, tumor necrosis factor-alpha (TNF- $\alpha$ ), interferon gamma (IFN- $\gamma)$.Upon this inflammatory response, proliferation and activation of antigenpresenting cells, including macrophages, histiocytes, and CD8+ T cells, promote the phagocytosis of other cells. HLH can be grouped as either primary (genetics) or secondary (acquired). The familial type has five known types, associated with various genetic mutations at various gene loci. In the acquired type of HLH, viral infections most commonly associated are Ebstein Barr, Herpes virus, Cytomegalovirus, Human immunodeficiency virus, influenza virus, rubella, Mycobacterium tuberculosis, Mycoplasma pneumoniae, fungi, and parasites (Kapoor et al., 2016). 
Once the diagnosis of HLH is established, the trigger factor should be identified, and treatment should be started immediately. In MAS, the treatment is with high dose steroids because it is associated more with juvenile rheumatoid arthritis (Hadchouel et al., 1985). HLH has the median survival of two months if left untreated. This patient had sarcoidosis with pancytopenia, high ferritin levels, and new onset of liver dysfunction despite treatment with high dose steroids and etoposide suggested HLH/MAS. There are no set guidelines for HLH treatment therefore aggressive treatment should be started once a preliminary diagnosis has been made. The HLH-94 protocol initially recommended starting treatment of eight weeks of induction therapy of dexamethasone, etoposide, and intrathecal methotrexate. Latest guidelines include involving cyclosporine in the beginning of the treatment, and then adding hydrocortisone to intrathecal methotrexate (Henter et al., 2007; Kleynberg and Schiller, 2012; Jordan et al., 2011).

Once the eight weeks are completed, patients are weaned off therapy and prepared for hematopoietic cell transplant. These guidelines were designed for patients younger than eighteen, or those with severe HLH.

\section{Conclusion}

In conclusion, the association between sarcoidosis and HLH is rare. This patient's worsening clinical status with steadily decreasing blood counts, high ferritin levels, liver involvement, and resistant to steroids, led to the diagnosis of HLH/MAS. Once HLH is diagnosed, it is imperative to be aggressive in treatment to salvage the patient. Further studies and trials should be performed on HLH cases in adults, which may aid in decreasing the mortality of the disease.

\section{References}

Abughanimeh O, Qasrawi A, Ghanimeh MA. Hemophagocytic Lymphohistiocytosis Complicating Systemic Sarcoidosis. Cureus 2018; 10: e2838.

Atteritano M, David A, Bagnato G, Beninati C, Frisina A, Iaria C, Bagnato G, Cascio A. Haemophagocytic syndrome in rheumatic patients. A systematic review. Eur Rev Med Pharmacol Sci 2012; 16: 1414-1424.

Hadchouel M, Prieur AM, Griscelli C. Acute hemorrhagic, hepatic, and neurologic manifestations in juvenile rheumatoid arthritis: possible relationship to drugs or infection. The Journal of pediatrics 1985; 106: 561-566.

Henter JI, Horne A, Aricó M, Egeler RM, Filipovich AH, Imashuku S, Ladisch S, McClain K, Webb D, Winiarski J, Janka G. HLH-2004: diagnostic and therapeutic guidelines for hemophagocytic lymphohistiocytosis. Pediatric blood \& cancer 2007; 48: 124-131.

Jordan MB, Allen CE, Weitzman S, Filipovich AH, McClain KL. How I treat hemophagocytic lymphohistiocytosis. Blood 2011; 118: 4041-4052. 
Global Journal of Cancer Case Reports | November 2019 | Volume 01| Issue 01 | PAGE 1-5

Patel D., A Rare Case of Hemophagocytic Lymphohistiocytosis with Concurrent Extra-Pulmonary Sarcoidosis Presenting as Fever of Unknown Origin

Kapoor R, Giri PP, Das J. Fulminant Familial Hemophagocytic Lymphohistiocytosis (FHL) in an Infant Masquerading Septic Shock-A case report. Journal of Pediatric Critical Care 2016: 152.

Kleynberg RL and Schiller GJ. Secondary hemophagocytic lymphohistiocytosis in adults: an update on diagnosis and therapy. Clin Adv Hematol Oncol 2012; 10: 726-732.

Rosado FG and Kim AS. Hemophagocytic LymphohistiocytosisAn Update on Diagnosis and Pathogenesis. American Journal of Clinical Pathology 2013; 139: 713-727.

Sawhney S, Woo P, Murray KJ. Macrophage activation syndrome: a potentially fatal complication of rheumatic disorders. Archives of disease in childhood 2001; 85: 421-426.

Schram AM and Berliner N. How I treat hemophagocytic lymphohistiocytosis in the adult patient. Blood 2015; 125: 2908-2914. 